\title{
Frontières
}

\section{VACHER, Laurent-Michel, Une petite fin du monde. Carnet devant la mort, Montréal, Liber, 2005, 197 p.}

\section{Jacques T. Godbout}

Volume 18, numéro 2, printemps 2006

URI : https://id.erudit.org/iderudit/1073228ar

DOI : https://doi.org/10.7202/1073228ar

Aller au sommaire du numéro

Éditeur(s)

Université du Québec à Montréal

ISSN

1180-3479 (imprimé)

1916-0976 (numérique)

Découvrir la revue

Citer ce compte rendu

Godbout, J. T. (2006). Compte rendu de [VACHER, Laurent-Michel, Une petite fin du monde. Carnet devant la mort, Montréal, Liber, 2005, 197 p.] Frontières,

18(2), 77-77. https://doi.org/10.7202/1073228ar

Ce document est protégé par la loi sur le droit d'auteur. L'utilisation des services d'Érudit (y compris la reproduction) est assujettie à sa politique d'utilisation que vous pouvez consulter en ligne.

https://apropos.erudit.org/fr/usagers/politique-dutilisation/
Cet article est diffusé et préservé par Érudit.

Érudit est un consortium interuniversitaire sans but lucratif composé de l’Université de Montréal, l’Université Laval et l’Université du Québec à Montréal. Il a pour mission la promotion et la valorisation de la recherche. https://www.erudit.org/fr/ 
VACHER, Laurent-Michel

\section{Une petite fin du monde}

\section{Carnet devant la mort}

Montréal, Liber,

2005, 197 p.

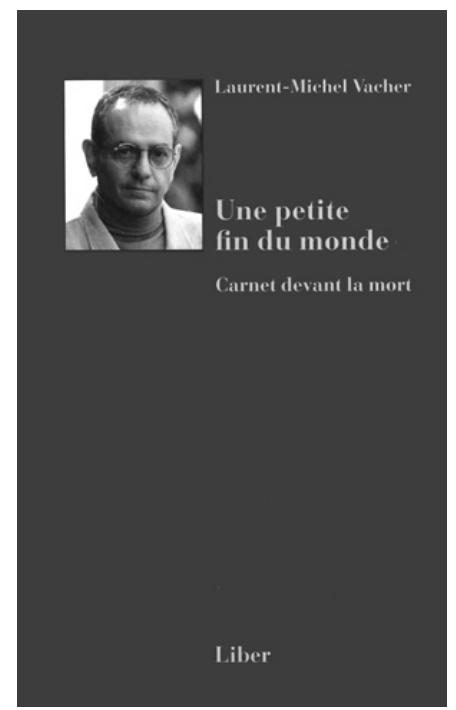

Philosophe pamphlétaire, grand défenseur de la philosophie des Lumières et de la science, partisan de la lucidité et pourfendeur des illusions, Laurent-Michel Vacher est décédé le 8 juillet 2005 d'un cancer du foie. Pendant les derniers mois de sa vie, il a écrit ce qu'il a appelé son carnet devant la mort. II nous expose avec conviction et émotion, mais sans pathos, les valeurs humanistes qu'il a toujours défendues. II y déploie ses dernières énergies à tenter de nous convaincre que I'univers n'est que matière. "La foi au surnaturel... cultive le sentiment de communion - et de soumission à une réalité supérieure englobante... qui n'existe pas» (p. 56). Toute personne raisonnable et rationnelle ne peut qu'arriver à cette conclusion. L'humanité est un phénomène émergent que la science expliquera, il disparaîtra un jour et personne n'en saura jamais rien. Toute pensée non matérialiste, toute "vision du monde comme mystère» (p. 65, citation de Alberoni) est une faiblesse, bien compréhensible, certes, mais une personne lucide se doit de rejeter de telles illusions, même et surtout devant l'annonce de sa propre mort prochaine.

"On ne nous a rien promis" (Alain). Le pamphlétaire n'est jamais très loin, même si le ton est calme et plein de respect pour ceux qui ne penseraient pas comme lui. C'est la dernière œuvre d'un «irréligieux militant» (p. 64). Si je n'étais pas devenu athée, j'aurais fait un curé, croit-il. «La perte de la foi religieuse a constitué dans ma vie un tournant déterminant. Je serais, sinon, devenu prêtre plutôt que philosophe, j'en ai peur» (p. 59).

Ce livre est un documentémouvant offrant un témoignage d'une mort digne. Mais je me permets de poser la question: cette position matérialiste est-elle bien la seule raisonnable et rationnelle? Au vu des conceptions scientifiques actuelles de l'univers, - le big-bang, les quantas, l'énergie noire -, n'y aurait-il pas au moins une autre attitude aussi raisonnable: I'incertitude? Ne serait-ce pas même la seule conclusion raisonnable qu'on puisse tirer des connaissances scientifiques actuelles? Ces connaissances s'éloignent toujours plus des certitudes réductionnistes que semblaient posséder les théories classiques et laissent ces questions ouvertes. Plus on s'éloigne de la simplicité de ces théories, plus on s'ouvre sur des perspectives inimaginables. «Ce n'est pas seulement que l'univers est plus étrange que nous l'imaginons. II est plus étrange que nous ne pouvons l'imaginer ", écrit l'astrophysicien qui a vérifié la théorie de l'espace courbe d'Einstein, Sir Arthur Eddington. Après tout, on vit un miracle quotidien permanent avec le phénomène de la gravitation. Nul ne sait encore avec certitude pourquoi, ultimement, les corps s'attirent. Dans un esprit matérialiste, Newton avait postulé l'éther. Car, affirmait-il, «il est inconcevable que la matière brute inanimée, sans la médiation d'autre chose qui ne soit pas matérielle, agisse sur une autre matière sans contact mutuel» (cité par Koestler, 1960, p. 483). Ce qui ne l'a pas empêché de mesurer cette force, en postulant une substance invisible reliant les choses entre elles à laquelle personne ne croit plus aujourd'hui.

Face aux grandes questions que l'homme ne peut pas ne pas se poser, la science ne répond rien. D'accord, elle remplace la Genèse par le big-bang, et ça fait quand même plus sérieux. Mais qu'y avait-il avant le big-bang? Un autre univers, non matériel? Un univers mathématique, comme l'affirment actuellement certains astrophysiciens? La science et les scientifiques l'ignorent, ils spéculent. Bref, plus la connaissance de l'univers progresse, plus la prétention de le connaître suffisamment pour se prononcer sur ces questions et sur l'existence d'autres sortes d'êtres apparaît... prétentieuse. Avant de se donner la mort, Arthur Koestler a écrit: "Je souhaite que mes amis sachent que je quitte leur compagnie en paix avec l'espoir ténu d'une aprèsvie dépersonnalisée au-delà des limites de l'espace, du temps et de la matière, et au-delà des limites de notre compréhension. » Cette attitude est-elle plus ou moins rationnelle que celle de L.-M. Vacher? Personnellement, il m'arrive de penser comme Koestler, et il m'arrive aussi, comme Vacher, d'avoir la conviction qu'il n'existe pas d'autres univers que le nôtre et que si jamais ils existent, ils ne sont faits que de matière eux aussi. J'oscille entre différentes attitudes sur ce sujet; je dois bien reconnaître qu'elles dépendent de mes états d'âme que l'expérience de la maladie ou de la mort d'êtres proches exercent une influence certaine sur ma vision du monde. Mais il me semble irrationnel de prétendre que l'une ou I'autre position est plus rationnelle que l'autre.

Le livre de Vacher parle beaucoup de religion, mais peu de spiritualité, et pas du tout des religions ou spiritualités orientales, telles que le bouddhisme qui, face à la mort, prône le lâcher prise, le détachement face au moi qui n'est que pure illusion. Nous, Occidentaux, arrivons mal à voir comment ce détachement peut en même temps s'accompagner de la compassion et de l'amour pour la vie comme le prône le bouddhisme. Peut-être est-ce seulement devant la mort que l'Occidental peut en arriver à concevoir une pareille vision de la vie... C'est du moins ce que la lecture de l'ouvrage de Vacher pourrait suggérer. Car lorsqu'on arrive à la dernière page de ces carnets devant la mort, écrite sans doute peu de temps avant le moment fatidique, la surprise n'est pas mince de constater que l'auteur se rapproche tout à coup de cette conception et semble même y adhérer. Pour lui, I'univers n'est plus que matériel puisqu'il souhaite "communier avec l'énigme de l'univers, se percevoir comme partie prenante du grand tout qui nous porte et nous emporte, adhérer à la magie du réel et la célébrer silencieusement » (p. 100). Tout se passe comme si, dans ses derniers moments, la luci- dité courageuse de Laurent-Michel Vacher lui avait apporté la sérénité et permis de lâcher prise, de s'abandonner à autre chose, "qui nous porte et nous emporte ». Peutêtre alors aurait-il été sensible à la pensée de Koestler.

J'espère atteindre, le jour venu, ce calme, cette réconciliation avec le monde, ce sentiment de faire partie de quelque chose qui me dépasse, quelle que soit cette chose, sentiment parfaitement raisonnable, mais sentiment que nous ne pouvons pas atteindre par notre volonté. Il émerge en nous comme l'être, peut-être, a un jour émergé du néant. II nous est donné.

Jacques T. Godbout 